\title{
A CD13 inhibitor, ubenimex, synergistically enhances the effects of anticancer drugs in hepatocellular carcinoma
}

\author{
MASAFUMI YAMASHITA ${ }^{1}$, HIROSHI WADA ${ }^{1}$, HIDETOSHI EGUCHI ${ }^{1}$, HISATAKA OGAWA ${ }^{1}$, \\ DAISAKU YAMADA ${ }^{1}$, TAKEHIRO NODA ${ }^{1}$, TADAFUMI ASAOKA ${ }^{1}$, KOICHI KAWAMOTO ${ }^{1}$, \\ KUNIHITO GOTOH $^{1}$, KOJI UMESHITA ${ }^{2}$, YUICHIRO DOKI ${ }^{1}$ and MASAKI MORI ${ }^{1}$ \\ ${ }^{1}$ Department of Gastroenterological Surgery; ${ }^{2}$ Division of Health Sciences, \\ Graduate School of Medicine, Osaka University, Osaka 565-0871, Japan
}

Received January 6, 2016; Accepted January 30, 2016

DOI: 10.3892/ijo.2016.3496

\begin{abstract}
Cancer stem cells (CSCs) were reported to be involved in resistance to chemo/radiation therapy. We previously reported that CD13 was both a marker of CSCs and a candidate therapeutic target in HCC. In the present study, we explored the antitumor effect of a combined therapy, where ubenimex, a CD13 inhibitor, was combined with conventional anticancer drugs, fluorouracil (5-FU), cisplatin (CDDP), doxorubicin (DXR) and sorafenib (SOR), and we elucidated the mechanism of these combination therapies. We evaluated changes in the expression of CD13 before and after treatment with anticancer drugs and with or without ubenimex in the human HCC cell lines HuH7 and PLC/PRF/5. The interactions between the anticancer drugs and ubenimex were determined with isobologram analyses. We analyzed cell cycle, apoptosis, and intracellular reactive oxygen species (ROS) levels to explore the mechanisms of the combination therapies. In both cell lines, the expression of CD13 increased after a 72-h exposure to each anticancer drug alone $(\mathrm{P}<0.05)$, and the expression of $\mathrm{CD} 13$ decreased with ubenimex administration $(\mathrm{P}<0.05)$. Isobologram analyses indicated that ubenimex had synergistic effects with 5-FU, CDDP and DXR, and an additive effect with SOR. Cell cycle analyses showed that ubenimex decreased the proportion of cells in G0/G1. Ubenimex enhanced the effects of 5-FU, CDDP and DXR by increasing apoptosis and intracellular ROS levels. In combination therapies, ubenimex synergistically enhanced the antitumor effects of 5-FU, CDDP and DXR on cell cycle regulation and apoptosis induction in HCC cell lines. The effects of ubenimex were due to increased intracellular ROS levels.
\end{abstract}

Correspondence to: Dr Hidetoshi Eguchi, Department of Gastroenterological Surgery, Graduate School of Medicine, Osaka University, 2-2, E-2, Yamadaoka, Suita, Osaka 565-0871, Japan E-mail: heguchi@gesurg.med.osaka-u.ac.jp

Key words: CD13 antigen, hepatocellular carcinoma, cancer stem cells, reactive oxygen species, ubenimex

\section{Introduction}

Hepatocellular carcinoma (HCC) is the third leading cause of cancer-related deaths worldwide (1). Many treatment options are available for patients with HCC, including surgical resection, local ablation therapy, chemoembolization, liver transplantation and molecular target therapy. Nevertheless, the prognosis of HCC remains poor, due to intrahepatic spread, postsurgical recurrence and chemoresistance (2-4).

Cancer stem cells (CSCs) have emerged as a potential cause of many malignant properties of tumors, including tumorigenicity, chemoradiation resistance, metastasis and tumor recurrence (5). CSCs are believed to share unique characteristics with normal stem cells; for example, they have the ability to self-renew and produce differentiated cells. Subsequent to the identification of CSCs in leukemia (6), CSCs have been reported in various solid tumors, including breast cancer, melanoma and colon cancer (7-9). In studies on HCC, the side population (SP) fraction, CD133, CD90, CD44 and epithelial cell adhesion molecules, were identified as CSC-specific markers (10-13). Many CSC markers have been shown to be associated with disease progression and outcome; however, no molecular target therapy for these markers has been developed.

CD13/Aminopeptidase N (APN) is a zinc-binding, type 2 transmembrane ectopeptidase $(150 \mathrm{kDa})$, which is expressed on various cell types, such as kidney, intestinal epithelium, liver, placenta and lung cells $(14,15)$. CD13 was first described as a marker for hematopoietic cells of myeloid origin. Recent studies have indicated that CD13 has various functions, including roles in inflammatory and immunological responses, signal transduction, antigen processing, neuropeptide and cytokine degradation, angiogenesis and extracellular matrix degradation (16,17). However, high CD13 expression levels have been detected in various solid tumors; additionally, CD13 was reported to be correlated with malignant behavior in colon, prostate and non-small cell lung cancers (18-20).

We previously reported that CD13 might be both a marker of CSCs and a candidate therapeutic target in HCC (21). We showed that CD13-positive cells exhibited strong chemoradiation resistance in vitro and in vivo. Moreover, $\mathrm{CD} 13$ expression protected cells from DNA damage by regulating the levels of 
reactive oxygen species (ROS). Inhibition of CD13 induced tumor cell apoptosis and resulted in tumor disruption, via blocking the ability of dormant CSCs to self-renew and re-initiate a tumor.

Ubenimex is a CD13/APN inhibitor. Ubenimex has been used as an adjuvant chemotherapy drug because of its function as an immunoenhancer in oncotherapy and reported to prolong the survival of patients with acute adult non-lymphocytic leukemia.

This drug was found to be cytotoxic to various tumor cell lines (22-24). We previously reported that combining ubenimex with 5-FU treatment, which is a ROS-inducing chemotherapy, improved liver cancer treatment (21).

In the systemic treatment of HCC, only sorafenib has been shown to provide overall survival benefit in a phase 3 randomized control trial (25). However, as a locoregional chemotherapy, some conventional cytotoxic agents were used in clinical practice. Transcatheter arterial chemoembolization (TACE) is the golden standard for the treatment of intermediate-stage HCC, and involves the administration of chemotherapeutic drugs, such as cisplatin or doxorubicin with or without lipiodol (26). In addition, we previously reported the efficacy of 5-fluorouracil arterial infusion + interferon therapy (FAIT) for advanced HCC (27). Thus, we thought that these conventional cytotoxic agents were worth trying to evaluate the effects of combination therapy with ubenimex.

In the present study, we explored the effects of ubenimex in combination with various anticancer drugs, which were used in the treatment of $\mathrm{HCC}$, and we elucidated the mechanism underlying the effects of these combinations.

\section{Materials and methods}

Cell culture. Two human liver cancer cell lines, HuH-7 and PLC/PRF/5, were obtained from the Japan Cancer Research Resources Bank (Tokyo, Japan). These cells were cultured and maintained in Dulbecco's modified Eagle's medium supplemented with $10 \%$ fetal bovine serum (FBS) and $500 \mu \mathrm{g} / \mathrm{ml}$ penicillin-streptomycin. Cells were incubated at $37^{\circ} \mathrm{C}$ in a humidified atmosphere containing $5 \% \mathrm{CO}_{2}$ in air.

Cells were treated with 5-fluorouracil (5-FU), cisplatin (CDDP), doxorubicin (DXR; Wako Pure Chemical Industries, Osaka, Japan), sorafenib (SOR; BioVision, Tucson, AZ, USA), and ubenimex (kindly supplied by Nihon Kayaku, Tokyo, Japan).

Flow cytometry for detecting expression of CSC markers. To analyze CD13 expression, cells were incubated with anticancer drugs with/without ubenimex $(100 \mu \mathrm{g} / \mathrm{ml})$ for 24,48 and $72 \mathrm{~h}$. At this concentration, ubenimex decreased the cell viability to $78.4 \%$ in $\mathrm{HuH} 7$ and $81.2 \%$ in PLC/PRF/5 for $72 \mathrm{~h}$. Next, cells were resuspended at $10^{6}$ cells $/ 100 \mu \mathrm{l}$ and incubated for $60 \mathrm{~min}$ at room temperature with an anti-CD13 mouse monoclonal antibody (Santa Cruz Biotechnology, Santa Cruz, CA, USA). After incubation, the samples were washed twice with phosphate-buffered saline (PBS) and resuspended in PBS containing $1 \%$ FBS. Labeled samples were analyzed with flow cytometry on a FACSAria II (BD Biosciences, San Jose, CA, USA). The cells were routinely sorted twice and re-analyzed for purity.
To evaluate the change of other CSC marker after the exposure to cytotoxic agents with/without ubenimex, an anti-CD44 mouse monoclonal antibody (Santa Cruz Biotechnology) was used as described above.

Cell growth inhibition assay. Growth inhibition was tested with the 3-(4,5-dimethylthiazol-2-yl)-2,5-diphenyl tetrazolium bromide (MTT) assay (Sigma-Aldrich, St. Louis, MO, USA). Cells were cultured in 96-well culture plates with various concentrations of anticancer drugs and ubenimex, alone or in combination. After 72-h incubations, $10 \mu \mathrm{l}(50 \mu \mathrm{g})$ of MTT was added to each well and incubated for $4 \mathrm{~h}$ at $37^{\circ} \mathrm{C}$. Next, the medium was removed, and $100 \mu 1$ of acid isopropanol was added to dissolve the resultant formazan crystals. Plate absorbance was measured in a microplate reader at $570 \mathrm{~nm}$, and absorbance at $650 \mathrm{~nm}$ was measured as reference. Results were expressed as the percentage of absorbance relative to untreated controls.

Assessment of combined drug effects with isobologram analysis. Isobologram analyses were used to determine whether the interactions between anticancer drugs and ubenimex were additive, synergistic, or antagonistic $(28,29)$. Dose-dependent effects were determined for each compound; for combinations, the dose of one compound was varied, and the dose of the other compound was fixed. Data points on the isobologram were evaluated according to their positions relative to the diagonal. The lower left region indicated synergism, falling on the diagonal line indicated additive effects, and the upper right region indicated antagonism.

The combination index (CI) provided a means to analyze the combined effects with a median-effect plot analysis. The CI was calculated according to the following formula: $\mathrm{CI}=$ $\left(d A / D_{30} A\right)+\left(d B / D_{30} B\right)$, where $D_{30} A$ is the concentration of drug A (ubenimex) required to produce $30 \%$ of the effect, and $\mathrm{dA}$ is the concentration of drug $\mathrm{A}$ required to produce $30 \%$ of the effect when combined with $\mathrm{dB}$. Similarly, $\mathrm{D}_{30} \mathrm{~B}$ is the concentration of drug $\mathrm{B}$ (anticancer drug) required to produce $30 \%$ of the effect, and $\mathrm{dB}$ is the concentration of drug B required to produce $30 \%$ of the effect when combined with dA. The CI values were defined as follows: $<0.8=$ synergism; from 0.8 to $1.2=$ additive effect $;$ and $>1.2$ $=$ antagonism.

Cell cycle analysis with propidium iodide staining. For cell cycle analysis, cells were incubated with each anticancer drug, alone or with ubenimex $(100 \mu \mathrm{g} / \mathrm{ml})$ for $48 \mathrm{~h}$, then fixed in $70 \%$ ethanol on ice. After centrifugation, cells were stained with $50 \mathrm{mg} / \mathrm{ml}$ propidium iodide (PI) solution (Dojindo Molecular Technologies, Kumamoto, Japan) and $0.1 \mathrm{mg} / \mathrm{ml}$ RNase A (Invitrogen). Stained cells were analyzed with flow cytometry on a FACSAria II. Each histogram was constructed with data from at least 20,000 events. Flow cytometric analyses were performed with the FlowJo software (Digital Biology, Tokyo, Japan).

Apoptosis analysis with Annexin $V$ staining. The Annexin V-FITC apoptosis detection kit (BD Biosciences) was used to detect cells undergoing apoptosis after anticancer treatment, according to the manufacturer's protocol. 


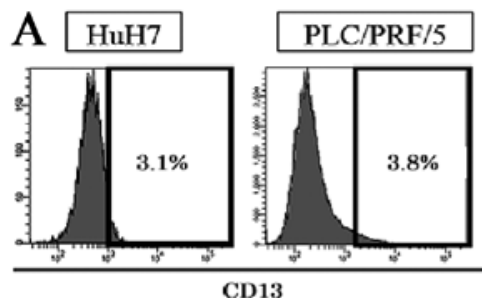

B $\mathrm{HuH7}$

5 -FU
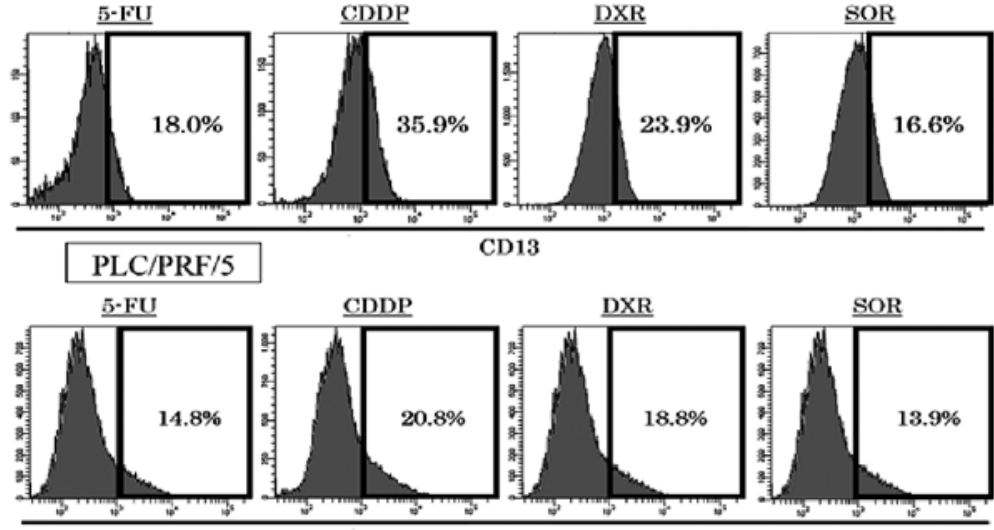

C $\mathrm{HuH7}$
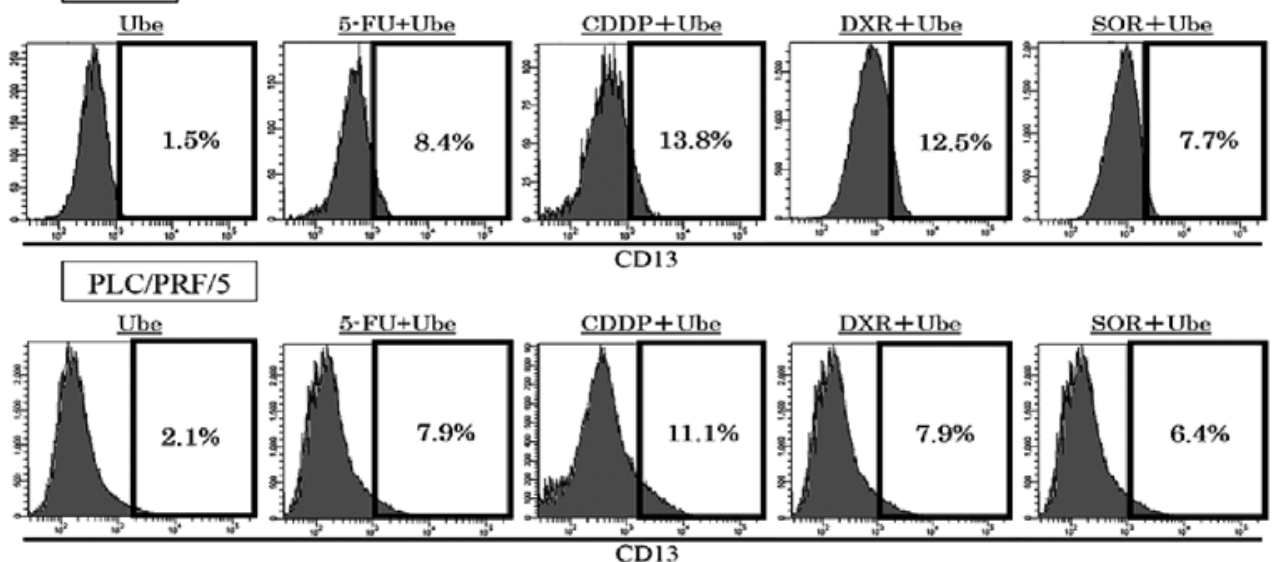

Figure 1. The expression of CD13 before and after treatment with anticancer drugs, with/without ubenimex. (A) The expression of CD13 was $3.5 \pm 0.5 \%$ in $\mathrm{HuH7}$ cells and $3.9 \pm 0.4 \%$ in PLC/PRF/5 cells. (B) The expression of CD13 increased after $72 \mathrm{~h}$ of exposure to 5-FU, CDDP, DXR and SOR in HuH7 cells and PLC/PRF/5 cells; $(\mathrm{P}<0.05)$. (C) When ubenimex (Ube) was combined with each anticancer drug, the expression of CD13 decreased compared to the anticancer drug alone in $\mathrm{HuH} 7$ cells and $\mathrm{PLC} / \mathrm{PRF} / 5$ cells $(\mathrm{P}<0.05)$.

Firstly, cells were incubated for $48 \mathrm{~h}$ with each anticancer drug or ubenimex $(100 \mu \mathrm{g} / \mathrm{ml})$ alone or in combination. Cell suspension $(100 \mu \mathrm{l})$ was mixed with $5 \mu \mathrm{l}$ of Annexin V-FITC and $2.5 \mu \mathrm{l}$ of PI and incubated for $30 \mathrm{~min}$ at room temperature in the dark. The samples were analyzed with flow cytometry on a FACSAria II (BD Biosciences). Cells stained with Annexin $\mathrm{V}$ were considered apoptotic cells.

Measurement of intracellular ROS levels. CellROX Deep Red reagent (Invitrogen, Gent, Belgium) is a fluorogenic probe for measuring intracellular oxidative stress in both live and fixed cells. The cell-permeant dye is nonfluorescent while in a reduced state and exhibits fluorescence upon oxidation by reactive oxygen species. After 48-h incubations with each anticancer drug, with/without ubenimex $(100 \mu \mathrm{g} / \mathrm{ml})$, cells were stained with $2 \mu \mathrm{M}$ CellROX Deep Red reagent by adding the probe to the complete medium and incubating the cells at $37^{\circ} \mathrm{C}$ for $30 \mathrm{~min}$ in the dark. Samples were analyzed with flow cytometry on a FACSAria II. The flow cytometric analysis was performed with FlowJo software.

Statistical analysis. Data are expressed as means \pm SD. The unpaired Student's t-test was used to examine differences between groups in cell proliferation, apoptosis, and cell cycle status. A P-value $<0.05$ was taken as statistically significant. Statistical analyses were performed with JMP Pro software, version 11.0 (SAS, Institute, Inc., Cary, NC, USA).

\section{Results}

The expression of CD13 increases with anticancer drugs and decreases with ubenimex. The expression of $\mathrm{CD} 13$ were assessed with FACS analyses in two human HCC cell lines. The expression of $\mathrm{CD} 13$ was $3.5 \pm 0.5 \%$ in $\mathrm{HuH} 7$ and $3.9 \pm 0.4 \%$ in PLC/PRF/5 (Fig. 1A). Exposure to ubenimex decreased the expression of $\mathrm{CD} 13 ; 1.5 \pm 0.3 \%$ in $\mathrm{HuH} 7,2.1 \% \pm 0.4 \%$ in 


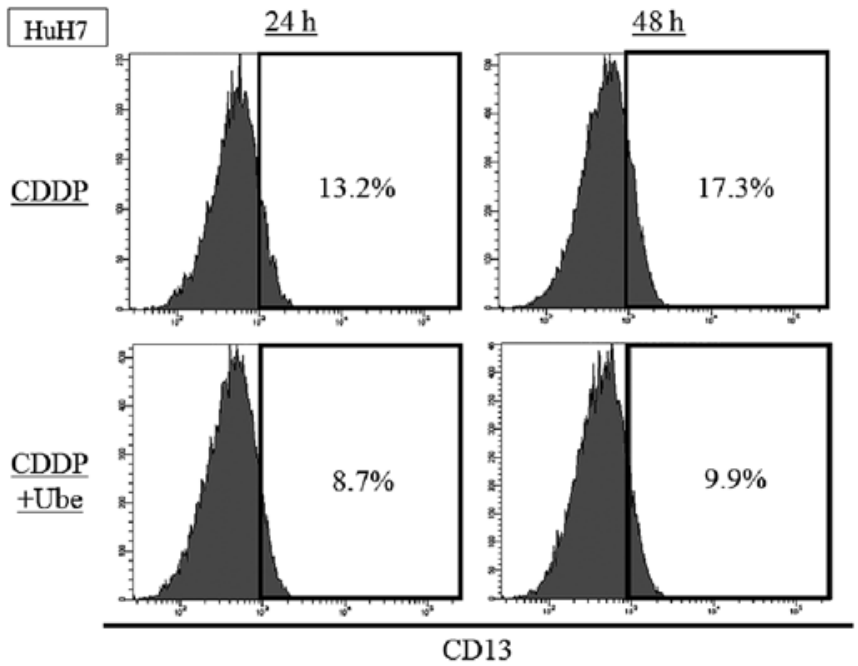

Figure 2. The expression of CD13 after 24- or 48-h CDDP treatment with/ without ubenimex. The expression of CD13 increased after 24- or 48-h CDDP treatment in $\mathrm{HuH} 7$ cells $(\mathrm{P}<0.05)$. When ubenimex was combined with CDDP, the expression of CD13 decreased compared to CDDP alone in HuH7 cells $(\mathrm{P}<0.05)$.

PLC/PRF/5. After 72-h exposure to each anticancer drug alone, the expression of CD13 restrictively increased; in $\mathrm{HuH7}$, expression of $\mathrm{CD} 13$ was $18.0 \pm 1.3 \%$ by 5 -FU treatment, $35.9 \pm 3.2 \%$ by CDDP treatment, $23.9 \pm 2.6 \%$ by DXR treatment, and $16.6 \pm 1.9 \%$ by SOR treatment; in PLC/PRF/5, $14.8 \pm 1.3 \%$ by 5 -FU treatment, $20.8 \pm 1.9 \%$ by CDDP treatment, $18.8 \pm 1.6 \%$ by DXR treatment, and $13.9 \pm 1.4 \%$ by SOR treatment $(\mathrm{P}<0.05$; Fig. 1B). When ubenimex was combined with each anticancer drug, the expression of CD13 increased less than that observed with the anticancer drugs alone, in both cell lines; in $\mathrm{HuH} 7$, the expression of $\mathrm{CD} 13$ decreased to $8.4 \pm 1.1 \%$ by 5 -FU treatment, $13.8 \pm 1.9 \%$ by CDDP treatment, $12.5 \pm 1.8 \%$ by DXR treatment, and $7.7 \pm 1.0 \%$ by SOR treatment; in PLC/PRF/5, $7.9 \pm 0.8 \%$ by 5-FU treatment, $11.1 \pm 0.9 \%$ by CDDP treatment, $7.9 \pm 0.8 \%$ by DXR treatment, and $6.4 \pm 0.5 \%$ by SOR treatment $(\mathrm{P}<0.05$; Fig. 1C).
We evaluated the expression of CD13 at earlier time-point. In $\mathrm{HuH} 7$, the expression of $\mathrm{CD} 13$ was $13.2 \pm 0.4 \%$ by $24-\mathrm{h}$ CDDP treatment and $17.3 \pm 1.1 \%$ by 48 -h CDDP treatment. When ubenimex was combined with CDDP, the expression of CD13 was decreased to $8.6 \pm 0.5 \%$ by 24 -h treatment and $9.9 \pm 0.8 \%$ by 48 -h treatment (Fig. 2 ).

The expression of CD44 increases by anticancer drugs and slightly decreases by ubenimex. To evaluate the change of other CSC markers after the exposure to cytotoxic agents with/without ubenimex, the expression of CD44 was assessed with FACS analysis in $\mathrm{HuH7}$. The expression of CD44 was $6.0 \pm 0.1 \%$, and the increase after exposure to CDDP; $18.7 \pm 0.5 \%$ at $24-\mathrm{h}$ and $19.9 \pm 0.3 \%$ at 48 -h. When ubenimex was combined with CDDP, the expression of CD44 slightly decreased relative to that observed with CDDP alone; $17.0 \pm 0.8 \%$ at 24 -h and $18.8 \pm 0.3 \%$ at 48 -h $(\mathrm{P}<0.05)$ (Fig. 3).

Interactions between ubenimex and anticancer drugs. The $\mathrm{IC}_{30}$ values of ubenimex alone was $394.8 \mu \mathrm{g} / \mathrm{ml}$ for $\mathrm{HuH} 7$, and $498.8 \mu \mathrm{g} / \mathrm{ml}$ for PLC/PRF/5. The MTT assay was performed with various concentrations of anticancer drugs combined with different fixed concentrations of ubenimex $(0,50,100$ and $200 \mu \mathrm{g} / \mathrm{ml}$; Fig. 4).

Isobologram analyses were performed, and CI values were calculated. The data points fell in the lower left region of the isobologram for combinations of ubenimex with 5-FU, CDDP and DXR (Fig. 3); all these CI values were $<0.8$ (Table I). The data points fell approximately on the diagonal for combinations of ubenimex with SOR, in both cell lines (Fig. 5); these CI values were between 0.8 and 1.2 in both cell lines (Table I).

The results indicated that, when ubenimex was combined with 5-FU, CDDP and DXR, the effect was synergistic, respectively, and combined with SOR, the effect was additive.

Ubenimex reduces the proportion of cells in $G 0 / G 1$ phase. We further explored the mechanism of synergistic effects in combination with ubenimex and anticancer drugs by examining the distribution of cells in different stages of the cell cycle. In both

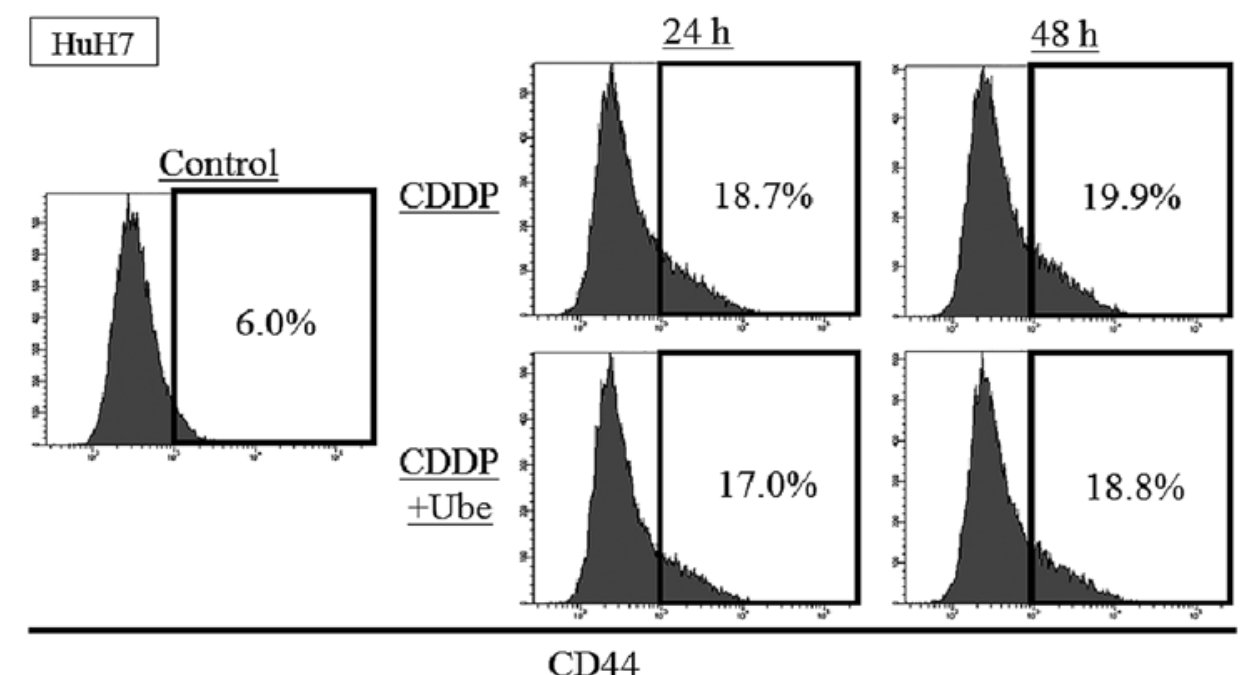

Figure 3. The expression of CD44 after 24- or 48-h CDDP treatment with/without ubenimex. The expression of CD44 was $6.0 \pm 0.1 \%$ in HuH7 cells. The expression of CD44 increased after 24- or 48-h CDDP treatment in HuH7 cells $(\mathrm{P}<0.05)$. When ubenimex was combined with CDDP, the expression of CD44 decreased compared to CDDP alone in $\mathrm{HuH7}$ cells $(\mathrm{P}<0.05)$. 


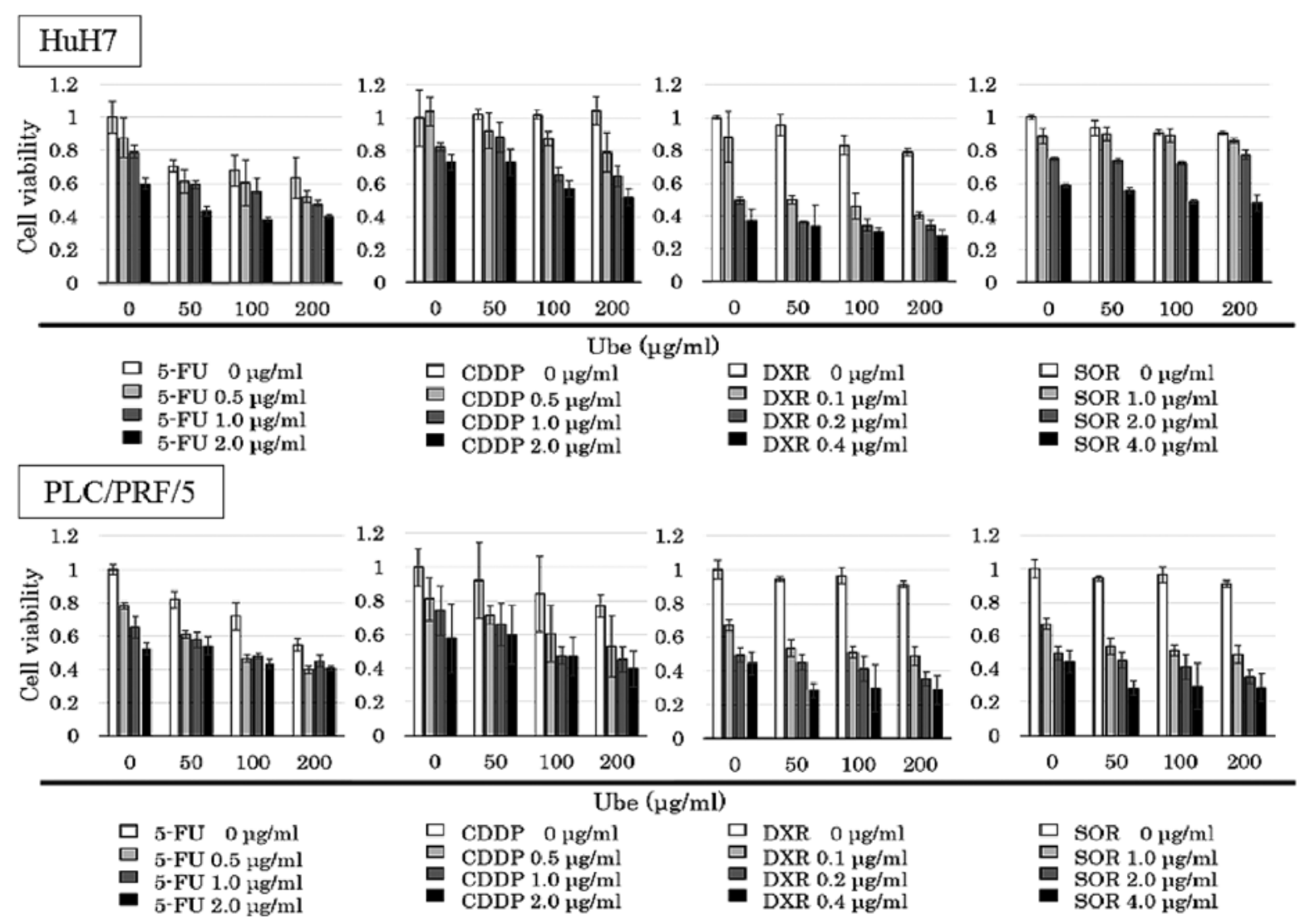

Figure 4. MTT assay results treated with or without ubenimex and cytotoxic agents. MTT assay results show viability of cells treated with various concentrations of anticancer drugs and ubenimex, alone or in combination for $72 \mathrm{~h}$ in HuH7 cells and PLC/PRF/5 cells.

Table I. The combination index for ubenimex combined with anticancer drugs.

\begin{tabular}{lcccc}
\hline Cell line & 5-FU & CDDP & DXR & SOR \\
\hline HuH7 & $0.49 \pm 0.36$ & $0.59 \pm 0.13$ & $0.68 \pm 0.13$ & $1.17 \pm 0.18$ \\
PLC/PRF/5 & $0.64 \pm 0.28$ & $0.59 \pm 0.16$ & $0.70 \pm 0.10$ & $0.92 \pm 0.10$ \\
\hline
\end{tabular}

5-FU, 5-fluorouracil; CDDP, cisplatin; DXR, doxorubicin; SOR, sorafenib. Data are expressed as mean $\pm \mathrm{SD}$.

cell lines, ubenimex alone did not affect the cell cycle. Cell lines treated with 5-FU, CDDP and DXR showed significantly smaller percentages of cells in the G0/G1 phase, and a larger percentage of cells in the $\mathrm{S} / \mathrm{G} 2 / \mathrm{M}$ phase, compared to control untreated conditions. In contrast, cells treated with SOR showed a larger percentage of cells in the G0/G1 phase and a smaller percentage of cells in the $\mathrm{S} / \mathrm{G} 2 / \mathrm{M}$ phases, compared to control conditions $(\mathrm{P}<0.05$ for each; Fig. 6). Moreover, in each cell line, when 5-FU, CDDP, DXR or SOR was combined with ubenimex, a significantly smaller percentage of cells were in the $\mathrm{G} 0 / \mathrm{G} 1$ phase and a larger percentage of cells were in the $\mathrm{S} / \mathrm{G} 2 / \mathrm{M}$ phase compared to when cells were exposed to the anticancer drug alone.

These results indicated that ubenimex reduced the proportion of cells in G0/G1 phase.

Ubenimex enhances apoptosis when combined with 5-FU, $C D D P$ and DXR. To quantify the percentage of cells under- going apoptosis, we performed the Annexin V assay (Fig. 7A). Cell lines treated with each anticancer drug alone showed a larger percentage of Annexin V-positive cells than that observed in control conditions. Moreover, when 5-FU, CDDP and DXR were combined with ubenimex, a higher percentage of Annexin V-positive cells was observed compared to that observed with the anticancer drugs alone $(\mathrm{P}<0.05)$. However, the percentage of Annexin $\mathrm{V}$-positive cells in cell lines treated with SOR alone was not significantly different from the percentage observed when SOR was combined with ubenimex (Fig. 7B).

Ubenimex increases the intracellular ROS when combined with 5-FU, CDDP and DXR. We evaluated the intracellular ROS levels when cells were treated with each anticancer drug, with or without ubenimex (Fig. 8A). Cell lines treated with each anticancer drug alone demonstrated an increase in ROS compared to control conditions. Additionally, in both cell lines, when 5-FU, CDDP or DXR was combined with ubenimex, the intracellular ROS increased above that observed with each drug alone $(\mathrm{P}<0.05)$. However, when SOR was combined with ubenimex, the intracellular ROS was not significantly different from that observed with SOR alone (Fig. 8B).

\section{Discussion}

CD13 is a transmembrane ectopeptidase to degrade peptides expressing on various organs and cell types. Previous studies also indicated that CD13 played an important role in control- 

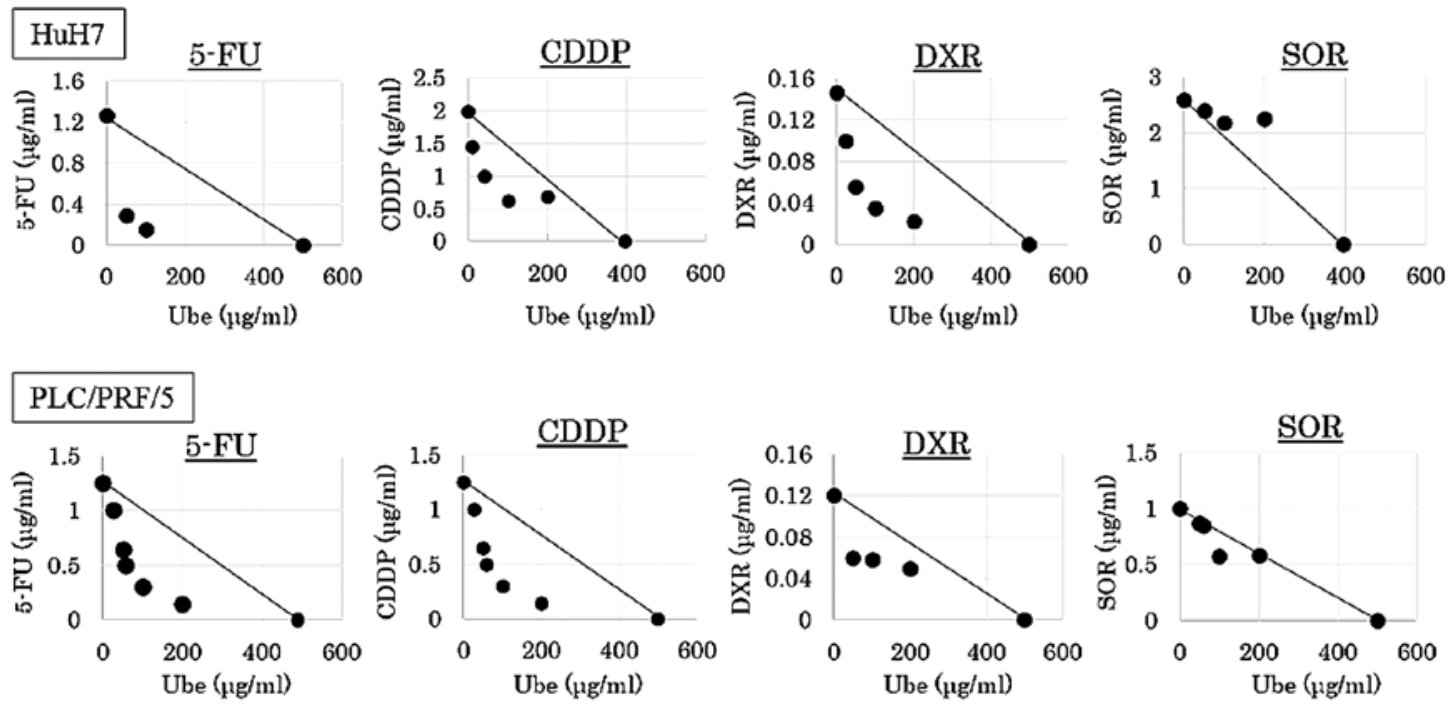

Figure 5. Interaction of ubenimex with cytotoxic agents. Isobologram based on the MTT assay results. Dots, data points from MTT assays; lines, dots located below, on, or above the line indicate synergistic, additive, or antagonistic effects, respectively.
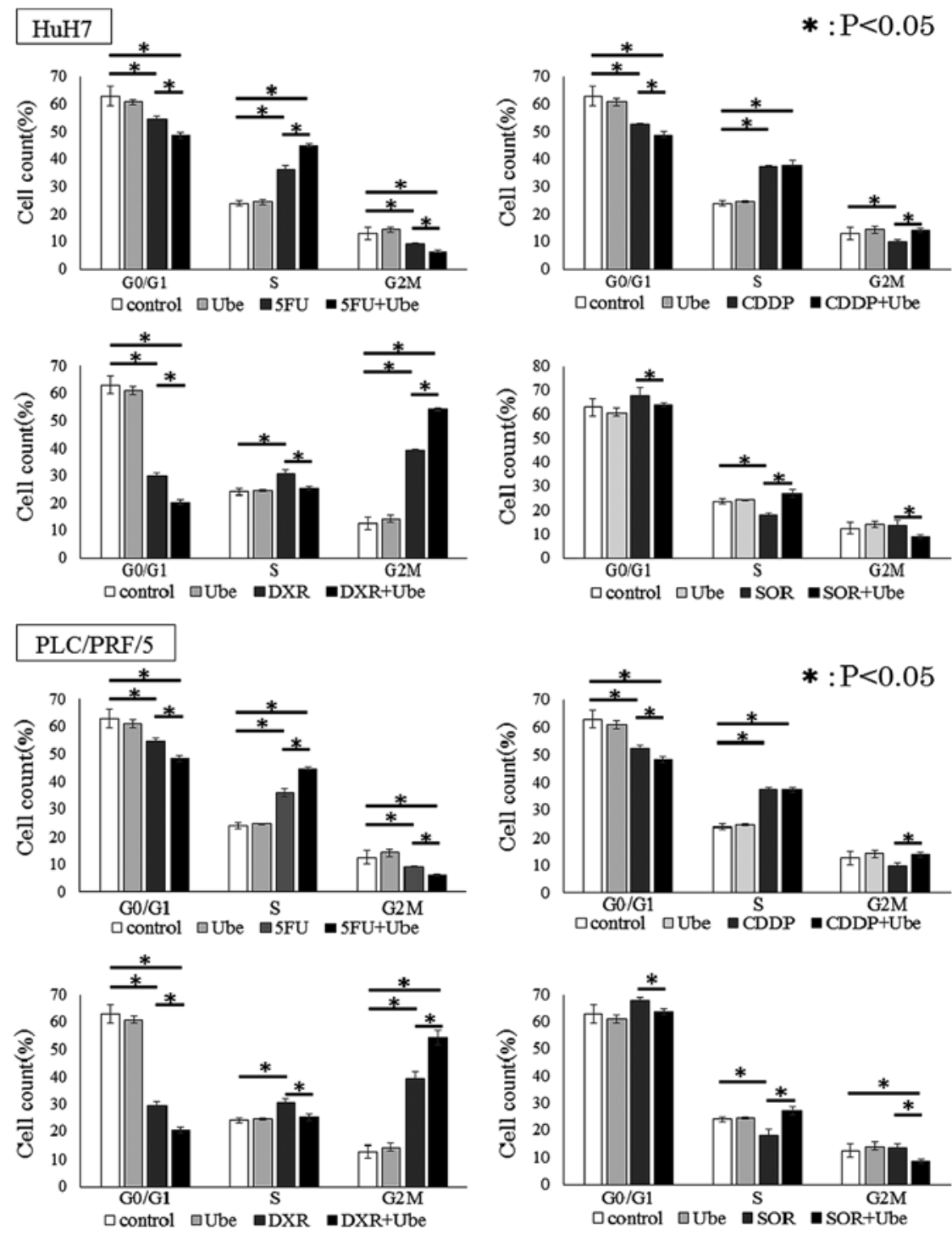

Figure 6. Ubenimex regulated cell cycle in cooperation with anticancer drugs. Compared to controls without drug (white), Ube (light gray) decreased the percentage of cells in the G0/G1 cell cycle phase. At 48 h, HuH7 cells and PLC/PRF/5 cells treated with 5-FU, CDDP, DXR and SOR combined with Ube (black) showed significantly smaller percentages of cells in the G0/G1 phase and larger percentages of cells in the S/G2/M phases, compared to the percentages observed with anticancer drugs alone (dark gray), (mean $\pm \mathrm{SD}$, from three different replicates) ${ }^{*} \mathrm{P}<0.05$. 

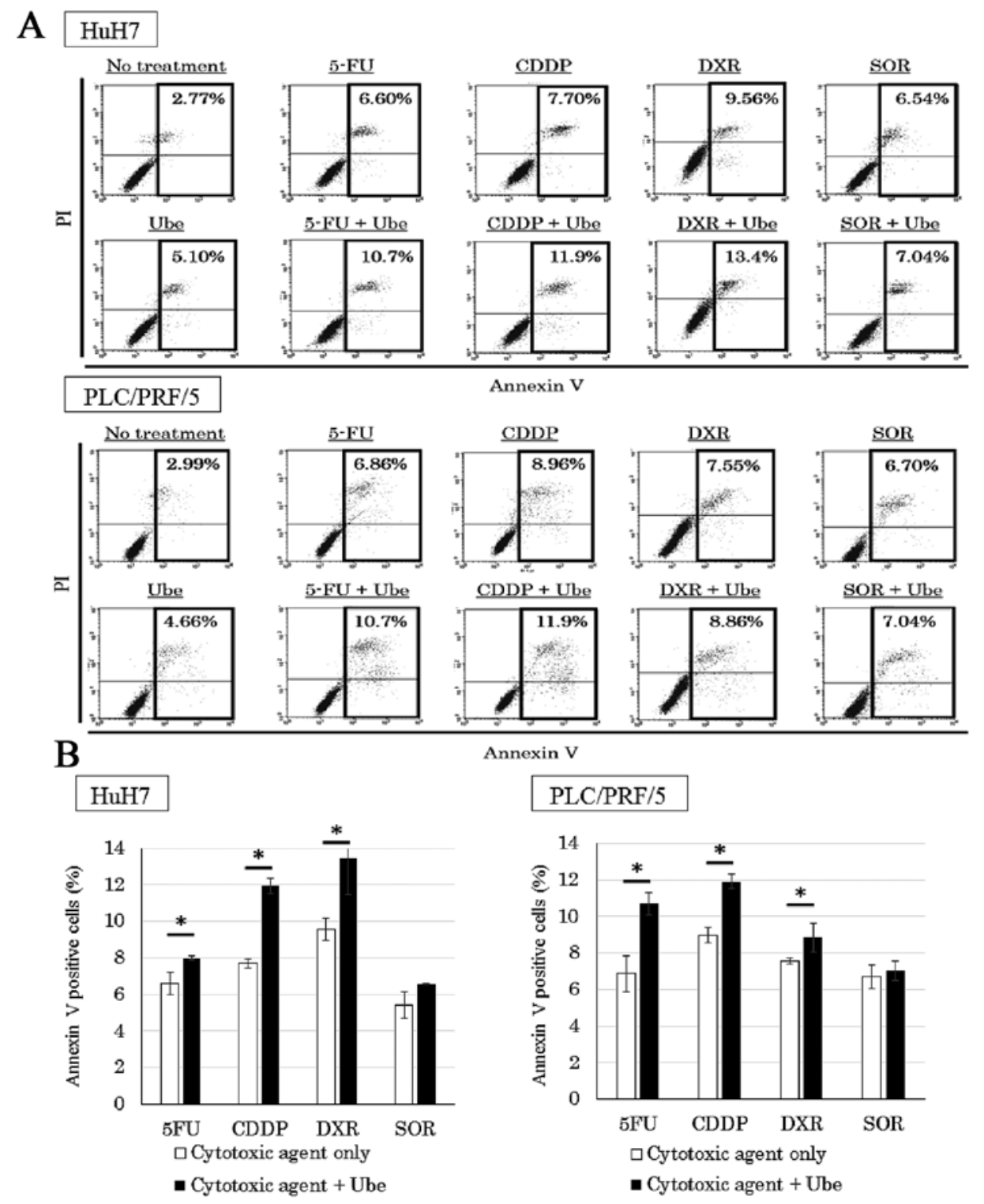

Figure 7. Ubenimex potentiated apoptosis when combined with 5-FU, CDDP and DXR, compared to the effects of each anticancer drug alone. Flow cytometric analyses with Annexin V assays showed that the rate of apoptosis was significantly higher in HuH7 cells and PLC/PRF/5 cells treated with 5-FU, CDDP and DXR combined with ubenimex (Ube), compared to those treated with anticancer drug alone. (A) The percentage of apoptotic cells detected (mean \pm SD, from three different replicates) (B), ${ }^{*} \mathrm{P}<0.05$.

ling cancer cell growth and differentiation. Inhibition of CD13 expression reduced proliferation in various types of cancer cells $(30,31)$. In addition, we previously demonstrated that CD13 was a CSC marker in HCC and a therapeutic target (21). In the present investigation, we examined the effect of combining ubenimex with conventional anticancer treatments, in vitro and in vivo.

The expression of CD13 increased by treatment with anticancer drug compared to untreated condition. From the fact that the expression of CD13 increased in time-dependent manner, the results suggested the increase of CD13 expression was the results of selection by chemotherapy. However, the expression of CD13 increased by 24-h exposure of the anticancer drug, so these results also indicate that anticancer drugs themselves upregulated CD13 expression. Ubenimex, which inhibited CD13 activity, reduced the number of CD13-positive cells by sensitizing cells to the anticancer drugs.
To evaluate the influence of ubenimex to other CSC markers, we examined the expression of CD44 after chemotherapy with ubenimex. The expression of CD44 increased by anticancer treatment. The combined therapy with ubenimex slightly decreased the expression of CD44, and the result of reduction ratio was statistically significant. The CSC marker positive cells included cancer stem cells and CD13-positive cells and CD44-positive cells could overlap each other partially. Ubenimex mainly reduces the expression of CD13 and might reduce the population of the overlapped CSC marker positive cells.

Isobologram analyses revealed that, when ubenimex was combined with 5-FU, CDDP and DXR, the effects were synergistic, and when combined with SOR, the effect was additive. To explore the mechanism underlying the synergistic effects, we focused on the cell cycle, apoptosis and intracellular ROS levels, because previous studies suggested that 
A $\mathrm{HuH7}$

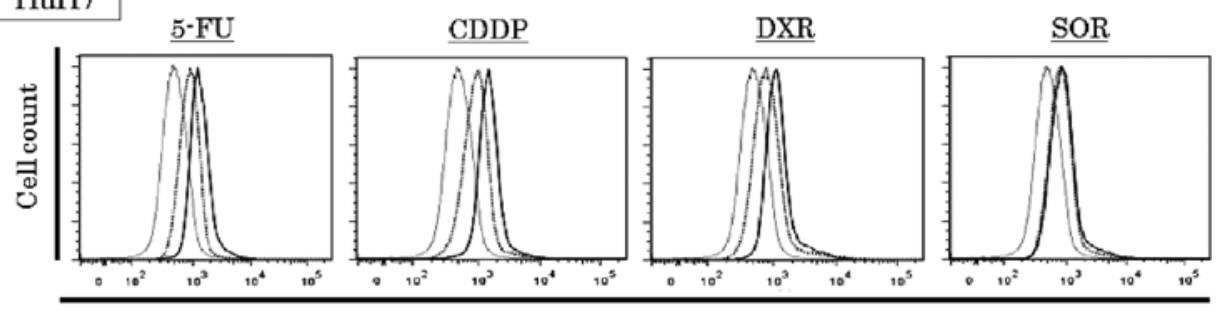

\section{$\mathrm{PLC} / \mathrm{PRF} / 5$}

CellROXfluorescence
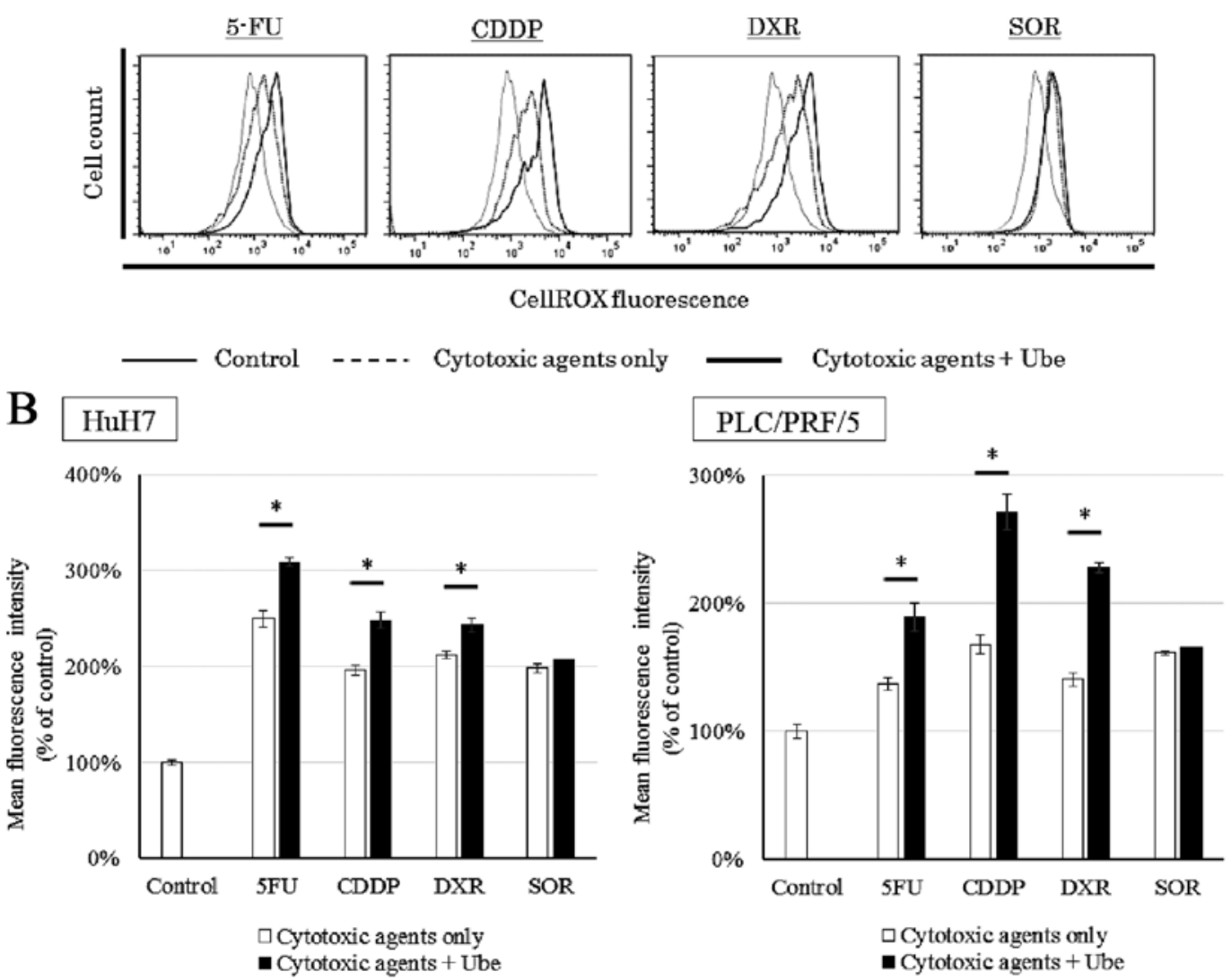

Figure 8. Ubenimex potentiates increased intracellular ROS levels when combined with 5-FU, CDDP and DXR, compared to the effects of each anticancer drug alone. HuH7 cells and PLC/PRF/5 cells treated with each anticancer drug alone (dotted lines) demonstrated an increase in ROS compared to control conditions (solid thin lines). When 5-FU, CDDP, and DXR were combined with ubenimex (solid bold lines), ROS detection (cellROX) increased compared to levels detected with each drug alone (dotted lines). (A) However, the ROS levels in cell lines treated with SOR alone were similar to ROS levels in cells treated with SOR combined with ubenimex. (B) The median of ROS-dependent fluorescence was detected (mean $\pm \mathrm{SD}$, from three different replicates), $\mathrm{P}<0.05$.

CSCs were resistant to various anticancer drugs, due to cell cycle dormancy, activated DNA-repair mechanisms, and low intracellular ROS levels (32).

The cell cycle analyses revealed that both 5-FU and CDDP alone could cause S-phase arrest or delay, and DXR alone caused G2/M-phase arrest. These results were consistent with previous reports (33-35). Ubenimex alone did not affect the cell cycle. However, when combined with anticancer drugs, the presence of ubenimex enhanced the cell cycle changes induced by the anticancer drugs. In the presence of ubenimex, 5-FU caused a greater increase in the proportion of cells in $\mathrm{S}$ phase, and DXR caused more cells to accumulate in G2M phase. These results suggested that ubenimex enhanced the effect of 5-FU on S-phase arrest and the effect of DXR on G2/M arrest. However, ubenimex did not enhance the effect of CDDP on S-phase accumulation. This result might be explained by the fact that platinum compounds mainly act on cancer cells by randomly damaging DNA, regardless of the cell cycle phase. The cell cycle analyses also revealed that ubenimex reduced the proportion of cells in G0/G1 phase after exposure to anticancer drugs. Our previous report demonstrated that the largest CD13-positive fraction was in the G0/G1 phase (21). These results indicated that inhibition of $\mathrm{CD} 13$ with ubenimex abrogated CD13-positive cell dormancy by inducing these cells to leave G0 and enter the cell cycle; this induction may sensitize CSCs to the effects of anticancer drugs.

We also showed that ubenimex alone induced apoptosis; moreover, combinations with 5-FU, CDDP, or DXR enhanced apoptosis compared to the effects of the anticancer drug alone. Several previous studies demonstrated that ubenimex induced cancer cell apoptosis by activating the pathway involving caspase-3, mitogen-activated protein kinase (MAPK), phos- 
phatidylinositol 3-kinase (PI3K), and glycogen synthase kinase-3 $\beta$ (GSK-3 $\beta)(22,36,37)$. However, the detailed mechanism of the induction has not been elucidated. We reasoned that ubenimex may induce apoptosis through its inhibition of CD13 activity; previously, CD13 was found to be involved in ROS excretion, and elevated ROS levels induced DNA oxidative damage and triggered apoptosis $(38,39)$.

ROS are oxygen-derived free radicals, such as hydroxyl radicals, peroxides and superoxides and are known to lead to DNA lesions, protein oxidation and lipid peroxidation (40). Currently, chemotherapeutic agents are used to cause DNA damage via ROS accumulation which leads to apoptosis induction in different tumor cells (41). The detailed mechanism of apoptosis via ROS formation remains to be clarified. For instance, some drugs have induced apoptosis through oxidative stress along with activation of the MAPK signaling pathways (42-44), that play an important role in the regulation of many cellular processes including cell growth and proliferation, differentiation, and apoptosis and downregulation of PI3K/AKT signaling pathways, that play a pivotal role in cell survival and the enhanced protection of cancer cells from apoptosis (45-47).

In our evaluations of intracellular ROS, ubenimex elevated intracellular ROS in combination with 5-FU, CDDP and DXR, but not with SOR. These results suggested that ROS played a key role in the synergistic effects of ubenimex on the mechanism of apoptosis induction. Also, they supported our hypothesis that 5-FU, CDDP and DXR induced DNA injury and increased ROS levels in CD13-positive cells, and that some of the increased ROS was excreted via CD13 (48-50). Therefore, inhibition of CD13 by ubenimex increased intracellular ROS levels in CD13-positive cells and induced apoptosis. Our findings suggested that only anticancer drugs that directly caused DNA damage and cell cycle entry would show synergistic effects when combined with ubenimex. Further studies are needed to determine the molecular mechanism of ubenimex.

Three of the tested cytotoxic agents showed synergism with ubenimex. However, SOR showed only an additive effect with ubenimex. SOR merely inhibits several tyrosine protein kinases (VEGFR and PDGFR) and Raf kinase (51); therefore, this drug did not directly induce DNA damage or cell cycle changes. Moreover, SOR was reported to be useful, both for treating HCC and for killing CSCs derived from HCC tumors (52). Although we demonstrated that exposure to SOR increased cellular CD13 expression, and that exposure to SOR combined with ubenimex decreased CD13 expression, SOR might affect HCC-derived CSCs through a different pathway. We previously demonstrated the effects of the combination therapy of 5-FU with ubenimex in vivo (21). Our results suggested that ubenimex may enhance the effect of transarterial chemoembolization using cisplatin or doxorubicin.

In conclusion, the present study demonstrated that combinations of 5-FU, CDDP and DXR with ubenimex synergistically enhanced their antitumor effects on cell cycle regulation and apoptosis induction, by increasing intracellular ROS levels in HCC cell lines. In clinical studies, ubenimex was shown to have beneficial effects in treatments for several types of malignancy, including leukemia, non-small cell lung and gastric cancer (53-55). Furthermore, our results provided novel insight into a chemotherapeutic strategy for HCC by adding ubenimex to chemotherapies currently in use.

\section{References}

1. Ferlay J, Shin HR, Bray F, Forman D, Mathers C and Parkin DM: Estimates of worldwide burden of cancer in 2008: GLOBOCAN 2008. Int J Cancer 127: 2893-2917, 2010.

2. Forner A, Llovet JM and Bruix J: Hepatocellular carcinoma. Lancet 379: 1245-1255, 2012.

3. Tomimaru Y, Wada H, Eguchi H, Tomokuni A, Hama N, Kawamoto K, Marubashi S, Umeshita K, Doki Y, Mori M, et al: Clinical significance of surgical resection of metastatic lymph nodes from hepatocellular carcinoma. Surg Today 45: 1112-1120, 2015.

4. Kobayashi T, Ishiyama $\mathrm{K}$ and Ohdan $\mathrm{H}$ : Prevention of recurrence after curative treatment for hepatocellular carcinoma. Surg Today 43: 1347-1354, 2013.

5. Valent P, Bonnet D, De Maria R, Lapidot T, Copland M, Melo JV, Chomienne C, Ishikawa F, Schuringa JJ, Stassi G, et al: Cancer stem cell definitions and terminology: The devil is in the details. Nat Rev Cancer 12: 767-775, 2012.

6. Bonnet D and Dick JE: Human acute myeloid leukemia is organized as a hierarchy that originates from a primitive hematopoietic cell. Nat Med 3: 730-737, 1997.

7. Al-Hajj M, Wicha MS, Benito-Hernandez A, Morrison SJ and Clarke MF: Prospective identification of tumorigenic breast cancer cells. Proc Natl Acad Sci USA 100: 3983-3988, 2003.

8. Fang D, Nguyen TK, Leishear K, Finko R, Kulp AN, Hotz S, Van Belle PA, Xu X, Elder DE and Herlyn M: A tumorigenic subpopulation with stem cell properties in melanomas. Cancer Res 65: 9328-9337, 2005.

9. O'Brien CA, Pollett A, Gallinger S and Dick JE: A human colon cancer cell capable of initiating tumour growth in immunodeficient mice. Nature 445: 106-110, 2007.

10. Ma S, Lee TK, Zheng BJ, Chan KW and Guan XY: CD133' HCC cancer stem cells confer chemoresistance by preferential expression of the Akt/PKB survival pathway. Oncogene 27: 1749-1758, 2008.

11. Yang ZF, Ho DW, Ng MN, Lau CK, Yu WC, Ngai P, Chu PW, Lam CT, Poon RT and Fan ST: Significance of CD90 cancer stem cells in human liver cancer. Cancer Cell 13: 153-166, 2008.

12. Yamashita T, Ji J, Budhu A, Forgues M, Yang W, Wang HY, Jia H, Ye Q, Qin LX, Wauthier E, et al: EpCAM-positive hepatocellular carcinoma cells are tumor-initiating cells with stem/progenitor cell features. Gastroenterology 136: 1012-1024, 2009.

13. Zhu Z, Hao X, Yan M, Yao M, Ge C, Gu J and Li J: Cancer stem/ progenitor cells are highly enriched in $\mathrm{CD} 133^{+} \mathrm{CD} 44^{+}$population in hepatocellular carcinoma. Int J Cancer 126: 2067-2078, 2010.

14. Stange T, Kettmann U and Holzhausen HJ: Immunoelectron microscopic single and double labelling of aminopeptidase $\mathrm{N}$ (CD 13) and dipeptidyl peptidase IV (CD 26). Acta Histochem 98: 323-331, 1996

15. Dixon J, Kaklamanis L, Turley H, Hickson ID, Leek RD, Harris AL and Gatter KC: Expression of aminopeptidase-n (CD 13) in normal tissues and malignant neoplasms of epithelial and lymphoid origin. J Clin Pathol 47: 43-47, 1994.

16. Bhagwat SV, Lahdenranta J, Giordano R, Arap W, Pasqualini R and Shapiro LH: CD13/APN is activated by angiogenic signals and is essential for capillary tube formation. Blood 97: 652-659, 2001.

17. Kehlen A, Egbert I, Thiele K, Fischer K, Riemann D and Langner J: Increased expression of interleukin-8 and aminopeptidase $\mathrm{N}$ by cell-cell contact: Interleukin- 8 is resistant to degradation by aminopeptidase N/CD13. Eur Cytokine Netw 12: 316-324, 2001.

18. Ishii K, Usui S, Sugimura Y, Yoshida S, Hioki T, Tatematsu M, Yamamoto $\mathrm{H}$ and Hirano $\mathrm{K}$ : Aminopeptidase $\mathrm{N}$ regulated by zinc in human prostate participates in tumor cell invasion. Int J Cancer 92: 49-54, 2001

19. Hashida H, Takabayashi A, Kanai M, Adachi M, Kondo K, Kohno N, Yamaoka Y and Miyake M: Aminopeptidase N is involved in cell motility and angiogenesis: Its clinical significance in human colon cancer. Gastroenterology 122: 376-386, 2002.

20. Tokuhara T, Hattori N, Ishida H, Hirai T, Higashiyama M, Kodama K and Miyake M: Clinical significance of aminopeptidase $\mathrm{N}$ in non-small cell lung cancer. Clin Cancer Res 12: 3971-3978, 2006. 
21. Haraguchi N, Ishii H, Mimori K, Tanaka F, Ohkuma M, Kim HM, Akita H, Takiuchi D, Hatano H, Nagano H, et al: CD13 is a therapeutic target in human liver cancer stem cells. J Clin Invest 120: 3326-3339, 2010.

22. Sekine K, Fujii H and Abe F: Induction of apoptosis by bestatin (ubenimex) in human leukemic cell lines. Leukemia 13: 729-734, 1999.

23. Ezawa K, Minato K and Dobashi K: Induction of apoptosis by ubenimex (Bestatin) in human non-small-cell lung cancer cell lines. Biomed Pharmacother 50: 283-289, 1996.

24. Terauchi M, Kajiyama H, Shibata K, Ino K, Nawa A, Mizutani S and Kikkawa F: Inhibition of APN/CD13 leads to suppressed progressive potential in ovarian carcinoma cells. BMC Cancer 7 : $140,2007$.

25. Llovet JM, Ricci S, Mazzaferro V, Hilgard P, Gane E, Blanc JF, de Oliveira AC, Santoro A, Raoul JL, Forner A, et al; SHARP Investigators Study Group: Sorafenib in advanced hepatocellular carcinoma. N Engl J Med 359: 378-390, 2008.

26. Lencioni R, Petruzzi P and Crocetti L: Chemoembolization of hepatocellular carcinoma. Semin Intervent Radiol 30: 3-11, 2013

27. Nagano H, Wada H, Kobayashi S, Marubashi S, Eguchi H, Tanemura M, Tomimaru Y, Osuga K, Umeshita K, Doki Y, et al: Long-term outcome of combined interferon- $\alpha$ and 5-fluorouracil treatment for advanced hepatocellular carcinoma with major portal vein thrombosis. Oncology 80: 63-69, 2011.

28. Chou TC and Talalay P: Quantitative analysis of dose-effect relationships: The combined effects of multiple drugs or enzyme inhibitors. Adv Enzyme Regul 22: 27-55, 1984.

29. Bijnsdorp IV, Giovannetti E and Peters GJ: Analysis of drug interactions. Methods Mol Biol 731: 421-434, 2011.

30. Wickström M, Larsson R, Nygren P, Gullbo J and Aminopeptidase N: Aminopeptidase N (CD13) as a target for cancer chemotherapy. Cancer Sci 102: 501-508, 2011.

31. Hitzerd SM, Verbrugge SE, Ossenkoppele G, Jansen G and Peters GJ: Positioning of aminopeptidase inhibitors in next generation cancer therapy. Amino Acids 46: 793-808, 2014.

32. Naka K, Muraguchi T, Hoshii T and Hirao A: Regulation of reactive oxygen species and genomic stability in hematopoietic stem cells. Antioxid Redox Signal 10: 1883-1894, 2008.

33. Maeda S, Wada H, Naito Y, Nagano H, Simmons S, Kagawa Y, Naito A, Kikuta J, Ishii T, Tomimaru Y, et al: Interferon- $\alpha$ acts on the $\mathrm{S} / \mathrm{G} 2 / \mathrm{M}$ phases to induce apoptosis in the G1 phase of an IFNAR2-expressing hepatocellular carcinoma cell line. J Biol Chem 289: 23786-23795, 2014

34. Qin LF and Ng IO: Induction of apoptosis by cisplatin and its effect on cell cycle-related proteins and cell cycle changes in hepatoma cells. Cancer Lett 175: 27-38, 2002.

35. Fan C, Zheng W, Fu X, Li X, Wong YS and Chen T: Strategy to enhance the therapeutic effect of doxorubicin in human hepatocellular carcinoma by selenocystine, a synergistic agent that regulates the ROS-mediated signaling. Oncotarget 5: 2853-2863, 2014.

36. Sawafuji K, Miyakawa Y, Weisberg E, Griffin JD, Ikeda Y and Kizaki M: Aminopeptidase inhibitors inhibit proliferation and induce apoptosis of K562 and STI571-resistant K562 cell lines through the MAPK and GSK-3beta pathways. Leuk Lymphoma 44: 1987-1996, 2003.

37. Liang W, Gao B, Xu G, Weng D, Xie M and Qian Y: Possible contribution of aminopeptidase N (APN/CD13) to migration and invasion of human osteosarcoma cell lines. Int J Oncol 45: 2475-2485, 2014

38. Prasad V, Chandele A, Jagtap JC, Sudheer Kumar P and Shastry P: ROS-triggered caspase 2 activation and feedback amplification loop in beta-carotene-induced apoptosis. Free Radic Biol Med 41: 431-442, 2006.

39. Madesh M, Zong WX, Hawkins BJ, Ramasamy $\mathrm{S}$, Venkatachalam T, Mukhopadhyay P, Doonan PJ, Irrinki KM, Rajesh M, Pacher P, et al: Execution of superoxide-induced cell death by the proapoptotic Bcl-2-related proteins Bid and Bak. Mol Cell Biol 29: 3099-3112, 2009.
40. Balaban RS, Nemoto S and Finkel T: Mitochondria, oxidants, and aging. Cell 120: 483-495, 2005.

41. Chetram MA, Bethea DA, Odero-Marah VA, Don-SaluHewage AS, Jones KJ and Hinton CV: ROS-mediated activation of AKT induces apoptosis via pVHL in prostate cancer cells. Mol Cell Biochem 376: 63-71, 2013.

42. McCubrey JA, Lahair MM and Franklin RA: Reactive oxygen species-induced activation of the MAP kinase signaling pathways. Antioxid Redox Signal 8: 1775-1789, 2006.

43. El-Najjar N, Chatila M, Moukadem H, Vuorela H, Ocker M, Gandesiri M, Schneider-Stock R and Gali-Muhtasib H: Reactive oxygen species mediate thymoquinone-induced apoptosis and activate ERK and JNK signaling. Apoptosis 15: 183-195, 2010

44. Navarro R, Busnadiego I, Ruiz-Larrea MB and Ruiz-Sanz JI: Superoxide anions are involved in doxorubicin-induced ERK activation in hepatocyte cultures. Ann NY Acad Sci 1090: 419-428, 2006.

45. Massaoka MH, Matsuo AL, Figueiredo CR, Farias CF, Girola N, Arruda DC, Scutti JA, Romoff P, Favero OA, Ferreira MJ, et al: Jacaranone induces apoptosis in melanoma cells via ROS-mediated downregulation of Akt and p38 MAPK activation and displays antitumor activity in vivo. PLoS One 7: e38698, 2012.

46. Yu CC, Wu PJ, Hsu JL, Ho YF, Hsu LC, Chang YJ, Chang HS, Chen IS and Guh JH: Ardisianone, a natural benzoquinone, efficiently induces apoptosis in human hormone-refractory prostate cancers through mitochondrial damage stress and survivin downregulation. Prostate 73: 133-145, 2013.

47. Hao W, Yuan X, Yu L, Gao C, Sun X, Wang D and Zheng Q: Licochalcone A-induced human gastric cancer BGC-823 cells apoptosis by regulating ROSmediated MAPKs and PI3K/AKT signaling pathways. Sci Rep 18: 5, 2015.

48. Hartmann KU and Heidelberger C: Studies on fluorinated pyrimidines. XIII. Inhibition of thymidylate synthetase. J Biol Chem 236: 3006-3013, 1961.

49. Wang D and Lippard SJ: Cellular processing of platinum anticancer drugs. Nat Rev Drug Discov 4: 307-320, 2005.

50. Lenaz L, Necco A, Dasdia T and Di Marco A: Biologic activity of some adriamycin (NSC-123127) derivatives. Cancer Chemother Rep 58: 769-776, 1974

51. Wilhelm SM, Carter C, Tang L, Wilkie D, McNabola A, Rong H, Chen C, Zhang X, Vincent P, McHugh M, et al: BAY 43-9006 exhibits broad spectrum oral antitumor activity and targets the RAF/MEK/ERK pathway and receptor tyrosine kinases involved in tumor progression and angiogenesis. Cancer Res 64: 7099-7109, 2004

52. Hashimoto N, Tsunedomi R, Yoshimura K, Watanabe Y, Hazama S and Oka M: Cancer stem-like sphere cells induced from de-differentiated hepatocellular carcinoma-derived cell lines possess the resistance to anti-cancer drugs. BMC Cancer 14: 722, 2014

53. Wakita A, Ohtake S, Takada S, Yagasaki F, Komatsu H, Miyazaki Y, Kubo K, Kimura Y, Takeshita A, Adachi Y, et al: Randomized comparison of fixed-schedule versus responseoriented individualized induction therapy and use of ubenimex during and after consolidation therapy for elderly patients with acute myeloid leukemia: The JALSG GML200 Study. Int J Hematol 96: 84-93, 2012.

54. Ichinose Y, Genka K, Koike T, Kato H, Watanabe Y, Mori T, Iioka S, Sakuma A and Ohta M; NK421 Lung Cancer Surgery Group: Randomized double-blind placebo-controlled trial of bestatin in patients with resected stage I squamous-cell lung carcinoma. J Natl Cancer Inst 95: 605-610, 2003.

55. Xu JW, Li CG, Huang XE, Li Y and Huo JG: Ubenimex capsule improves general performance and chemotherapy related toxicity in advanced gastric cancer cases. Asian Pac J Cancer Prev 12: 985-987, 2011. 